\title{
A WELL-POSED BOUNDARY VALUE PROBLEM FOR SUPERCRITICAL FLOW \\ OF VISCOELASTIC FLUIDS OF MAXWELL TYPE
}

\author{
By
}

Michael Renardy

IMA Preprint Series \# 535

June 1989 
This paper is dedicated to Daniel D. Joseph

on the occasion of his 60th birthday

\title{
A WELL-POSED BOUNDARY VALUE PROBLEM FOR SUPERCRITICAL FLOW OF VISCOELASTIC FLUIDS OF MAXWELL TYPE
}

\author{
MICHAEL RENARDY*
}

\begin{abstract}
For a class of viscoelastic fluids with differential constitutive laws of Maxwell type, we investigate the existence and uniqueness of steady flows. We consider small perturbations of uniform flow transverse to a strip. A well-posed boundary value problem is formulated for the case when the velocity of the fluid exceeds the speed of propagation of shear waves.
\end{abstract}

Key words. viscoelastic fluids, boundary conditions, change of type

AMS(MOS) subject classifications. 35M05,76A10

1. Introduction. While the study of existence and uniqueness results for steady flows of Newtonian fluids is well advanced, relatively little is known about viscoelastic fluids with memory. For such fluids, the nature of boundary conditions leading to well-posed problems is in general different from the Newtonian case. There are two reasons for this:

1. The memory of the fluid implies that what happens in the domain under consideration is dependent on the deformation history of the fluid before it entered the domain. Information about this deformation history must therefore be given in the form of boundary conditions at inflow boundaries. The precise nature of such inflow conditions is dependent on the constitutive relation; for example, fluids of Maxwell type [4] are different from fluids of Jeffreys type [5].

2. For fluids of Maxwell type, there is a change of type in the governing equations when the velocity of the fluid exceeds the propagation speed of shear waves (cf. [1], [2], [7], [8]). This necessitates a change in the nature of boundary conditions. If boundary conditions which would be correct in the subcritical case are imposed in a supercritical situation, the problem becomes ill-posed in a similar fashion as the Dirichlet problem for the wave equation (see [6]).

In the following, $v$ denotes the velocity, $p$ the pressure, $\mathbf{T}$ the extra stress tensor, $\rho$ the density and $f$ a given body force. The equation of motion is

$$
\rho(v \cdot \nabla) v=\operatorname{div} \mathbf{T}-\nabla p+f
$$

*Department of Mathematics and ICAM, Virginia Tech, Blacksburg, VA 24061-0123. This research was completed while I was visiting the Institute for Mathematics and its Applications at the University of Minnesota. Financial support from the IMA and from the National Science Foundation under Grant No. DMS-8796241 is gratefully acknowledged. 
and the incompressibility condition is

$$
\operatorname{div} v=0 .
$$

We assume a Maxwell-type constitutive relation of the following form:

$$
\begin{gathered}
((v \cdot \nabla)+\lambda) T_{i j}-\frac{\partial v_{i}}{\partial x_{k}} T_{k j}- \\
-T_{i k} \frac{\partial v_{j}}{\partial x_{k}}+P_{i k}(\mathbf{T})\left(\frac{\partial v_{k}}{\partial x_{j}}+\frac{\partial v_{j}}{\partial x_{k}}\right)+P_{j k}(\mathbf{T})\left(\frac{\partial v_{k}}{\partial x_{i}}+\frac{\partial v_{i}}{\partial x_{k}}\right) \\
+g_{i j}(\mathbf{T})=\mu\left(\frac{\partial v_{i}}{\partial x_{j}}+\frac{\partial v_{j}}{\partial x_{i}}\right) .
\end{gathered}
$$

Here $\lambda$ and $\mu$ are positive constants, and the matrix-valued functions $\mathbf{P}$ and $\mathbf{g}$ are assumed to be smooth; moreover, $\mathbf{P}, \mathbf{g}$ and the first derivatives of $\mathbf{g}$ vanish at $\mathbf{T}=\mathbf{0}$. Equation (3) includes a number of popular rheological models (cf. e.g. [3]).

The domain on which we want to solve (1)-(3) is the strip bounded by the planes $x_{1}=0$ and $x_{1}=1$. In the $x_{2}$ - and $x_{3}$-directions, we assume periodicity with periods $L$ and $M$. The solutions we seek are small perturbations of the uniform flow $v=(V, 0,0)$, $p=0, \mathbf{T}=0$. The given body force and the imposed boundary conditions are assumed to satisfy smallness conditions consistent with this. In [4], we considered this problem under the assumption that $\rho V^{2}<\mu$. A well-posed boundary value problem was obtained by prescribing the velocities at both boundaries plus additional stress conditions at the inflow boundary $x_{1}=0$. In two dimensions it is possible to prescribe the diagonal components $T_{11}$ and $T_{22}$. In three dimensions a correct choice of inflow stress conditions was obtained as follows. We expand each stress component in a Fourier series, e.g.

$$
T_{11}\left(0, x_{2}, x_{3}\right)=\sum_{k, l} t_{11}^{k l} \exp \left(2 \pi i\left(k x_{2} / L+l x_{3} / M\right)\right)
$$

Then one can, for example, prescribe the following inflow conditions:

$$
\begin{gathered}
t_{11}^{k l}, t_{22}^{k l}, t_{13}^{k l}, t_{33}^{k l} \text { if }|k|>>|l|, \\
t_{11}^{k l}, t_{22}^{k l}, t_{12}^{k l}, t_{33}^{k l} \text { if }|l|>>|k|, \\
t_{11}^{k l}, t_{13}^{k l}, t_{23}^{k l}, t_{33}^{k l} \text { if }|k| \text { and }|l| \text { are comparable, } \\
t_{11}^{k l}, t_{22}^{k l}, t_{23}^{k l}, t_{33}^{k l} \text { if } k=l=0 .
\end{gathered}
$$

If, on the other hand, $\rho V^{2}>\mu$, then this choice of boundary conditions does not lead to a well-posed problem [6]. We shall show that, in this case, one can prescribe the following conditions: the inflow stresses as above, the normal velocity at both boundaries, plus the vorticity and its normal derivative (in two dimensions), or, respectively, the second and third components of the vorticity and their normal derivatives (in three dimensions) at the inflow boundary. The analysis for two space dimensions will be carried out in Section 2; the modifications needed for three dimensions will be discussed in Section 3. 
2. The two-dimensional case. We apply the operation $(v \cdot \nabla)+\lambda+(\nabla v)^{T}$ to the equation of motion (1) and we use equation (3) to reexpress $((v \cdot \nabla)+\lambda) \mathbf{T}$. After some algebra, this yields an equation of the following form (written in components)

$$
\begin{gathered}
\rho((v \cdot \nabla)+\lambda)(v \cdot \nabla) v_{i}=\mu \Delta v_{i}+\left(T_{k j}-P_{k j}(\mathbf{T})\right) \frac{\partial^{2} v_{i}}{\partial x_{j} \partial x_{k}} \\
-P_{i k}(\mathbf{T}) \frac{\partial^{2} v_{k}}{\partial x_{j}^{2}}-P_{j k}(\mathbf{T}) \frac{\partial^{2} v_{k}}{\partial x_{i} \partial x_{j}}-\frac{\partial q}{\partial x_{i}} \\
+((v \cdot \nabla)+\lambda) f_{i}+\frac{\partial v_{j}}{\partial x_{i}} f_{j}+h_{i}(v, \nabla v, \mathbf{T}, \nabla \mathbf{T}) .
\end{gathered}
$$

Here we have set $q=((v \cdot \nabla)+\lambda) p$. The term $h$ is a complicated expression which we do not write out explicitly; it contains only quadratic and higher order terms.

Next we introduce a streamfunction-vorticity formulation. We set

$$
v_{1}=-\frac{\partial \psi}{\partial x_{2}}, v_{2}=\frac{\partial \psi}{\partial x_{1}}, \zeta=\frac{\partial v_{2}}{\partial x_{1}}-\frac{\partial v_{1}}{\partial x_{2}},
$$

so that the incompressibility condition (2) is automatically satisfied and

$$
\Delta \psi=\zeta .
$$

We take the curl of equation (6), which results in

$$
\begin{gathered}
\rho((v \cdot \nabla)+\lambda)(v \cdot \nabla) \zeta=\mu \Delta \zeta+\left(T_{k j}-P_{k j}(\mathbf{T})\right) \frac{\partial^{2} \zeta}{\partial x_{j} \partial x_{k}}-P_{22}(\mathbf{T}) \frac{\partial^{2} \zeta}{\partial x_{1}^{2}}-P_{11}(\mathbf{T}) \frac{\partial^{2} \zeta}{\partial x_{2}^{2}} \\
+\left(P_{12}(\mathbf{T})+P_{21}(\mathbf{T})\right) \frac{\partial^{2} \zeta}{\partial x_{1} \partial x_{2}}+r\left(v, \nabla v, \nabla^{2} v, \mathbf{T}, \nabla \mathbf{T}, \nabla^{2} \mathbf{T}, f, \nabla f, \nabla^{2} f\right) .
\end{gathered}
$$

Here $r$ is again a complicated expression which we do not write out explicitly.

In the following, we shall solve (1)-(3) subject to the following boundary conditions:

$$
\begin{gathered}
\psi=-V x_{2}+\psi_{0}, \text { on } x_{1}=0, \psi=-V x_{2}+\psi_{1}, \text { on } x_{1}=1, \\
\zeta=\zeta_{0}, \text { on } x_{1}=0, \frac{\partial \zeta}{\partial x_{1}}=\eta_{0}, \text { on } x_{1}=0, \\
T_{11}=t_{1}, \text { on } x_{1}=0, T_{22}=t_{2} \text { on } x_{1}=0 .
\end{gathered}
$$

We note that prescribing $\psi$ on both boundaries is equivalent to prescribing the normal velocity on these boundaries as well as the total flow rate in the $x_{2}$-direction.

We denote by $H^{s}$ the space of all functions on the strip $0 \leq x_{1} \leq 1$ which are periodic with period $L$ in the $x_{2}$-direction and have $s$ derivatives which are square integrable over one period. Sobolev spaces of periodic functions depending only on $x_{2}$ are denoted by $H^{\langle s\rangle}$. The corresponding norms are denoted by $\|\cdot\|_{s}$ and $\|\cdot\|_{\langle s\rangle}$. Moreover, $\|\cdot\|_{k, l}$ denotes the norm in $W^{k, \infty}\left([0,1] ; H^{\langle l\rangle}\right)$.

The goal of this section is the following existence and uniqueness result: 
Theorem. Assume that $\|f\|_{4},\left\|\psi_{0}\right\|_{\langle 9 / 2\rangle},\left\|\psi_{1}\right\|_{\langle 9 / 2\rangle},\left\|\zeta_{0}\right\|_{\langle 3\rangle},\left\|\eta_{0}\right\|_{\langle 2\rangle},\left\|t_{1}\right\|_{\langle 3\rangle}$ and $\left.\left\|t_{2}\right\|_{\langle 3\rangle}\right\rangle$ are sufficiently small. Then there is a solution of (1)-(3) which satisfies the boundary conditions (10) and the regularity $\psi+V x_{2} \in H^{5}, \mathbf{T} \in H^{3}$. Moreover, this solution is the only one for which $\left\|\psi+V x_{2}\right\|_{5}$, and $\|\mathbf{T}\|_{3}$ are small.

The construction of the solution is based on an iterative scheme. As a starting value for the iteration we use the uniform flow

$$
\psi^{0}=-V x_{2}, \zeta^{0}=0, \mathbf{T}^{0}=0 .
$$

Given $\psi^{n}, \zeta^{n}$ and $\mathbf{T}^{n}$, we define $v^{n}$ by

$$
v_{1}^{n}=-\frac{\partial \psi^{n}}{\partial x_{2}}, v_{2}^{n}=\frac{\partial \psi^{n}}{\partial x_{1}} .
$$

Next, we determine $\mathbf{T}^{n+1}$ from the equation

$$
\begin{gathered}
\left(\left(v^{n} \cdot \nabla\right)+\lambda\right) T_{i j}^{n+1}-\frac{\partial v_{i}^{n}}{\partial x_{k}} T_{k j}^{n}-T_{i k}^{n} \frac{\partial v_{j}^{n}}{\partial x_{k}}+P_{i k}\left(\mathbf{T}^{n}\right)\left(\frac{\partial v_{k}^{n}}{\partial x_{j}}+\frac{\partial v_{j}^{n}}{\partial x_{k}}\right)+P_{j k}\left(\mathbf{T}^{n}\right)\left(\frac{\partial v_{k}^{n}}{\partial x_{i}}+\frac{\partial v_{i}^{n}}{\partial x_{k}}\right) \\
+g_{i j}\left(\mathbf{T}^{n}\right)=\mu\left(\frac{\partial v_{i}^{n}}{\partial x_{j}}+\frac{\partial v_{j}^{n}}{\partial x_{i}}\right),
\end{gathered}
$$

subject to the following initial conditions at $x_{1}=0$ :

$$
\begin{gathered}
T_{11}^{n+1}=t_{1}, T_{22}^{n+1}=t_{2}, \\
\rho \operatorname{curl}\left(\left(v^{n} \cdot \nabla\right) v^{n}\right)=\operatorname{curl}\left(\operatorname{div} \mathbf{T}^{n+1}+f\right),
\end{gathered}
$$

Then we determine $\zeta^{n+1}$ from the initial-value problem

$$
\begin{gathered}
\rho\left(\left(v^{n} \cdot \nabla\right)+\lambda\right)\left(v^{n} \cdot \nabla\right) \zeta^{n+1}=\mu \Delta \zeta^{n+1}+\left(T_{k j}^{n+1}-P_{k j}\left(\mathbf{T}^{n+1}\right)\right) \frac{\partial^{2} \zeta^{n+1}}{\partial x_{j} \partial x_{k}} \\
-P_{22}\left(\mathbf{T}^{n+1}\right) \frac{\partial^{2} \zeta^{n+1}}{\partial x_{1}^{2}}-P_{11}\left(\mathbf{T}^{n+1}\right) \frac{\partial^{2} \zeta^{n+1}}{\partial x_{2}^{2}}+\left(P_{12}\left(\mathbf{T}^{n+1}\right)+P_{21}\left(\mathbf{T}^{n+1}\right)\right) \frac{\partial^{2} \zeta^{n+1}}{\partial x_{1} \partial x_{2}} \\
+r\left(v^{n}, \nabla v^{n}, \nabla^{2} v^{n}, \mathbf{T}^{n+1}, \nabla \mathbf{T}^{n+1}, \nabla^{2} \mathbf{T}^{n+1}, f, \nabla f, \nabla^{2} f\right) . \\
\zeta^{n+1}=\zeta_{0}, \text { on } x_{1}=0, \frac{\partial \zeta^{n+1}}{\partial x_{1}}=\eta_{0}, \text { on } x_{1}=0 .
\end{gathered}
$$


Finally, we obtain $\psi^{n+1}$ from the Dirichlet problem

$$
\begin{gathered}
\Delta \psi^{n+1}=\zeta^{n+1}, \\
\psi^{n+1}=-V x_{2}+\psi_{0}, \text { on } x_{1}=0, \psi^{n+1}=-V x_{2}+\psi_{1} \text {, on } x_{1}=1 .
\end{gathered}
$$

We define

$$
X(M)=\left\{(\psi, \zeta, \mathbf{T}) \mid\left\|\psi+V x_{2}\right\|_{5}+\|\zeta\|_{3}+\|\mathbf{T}\|_{3} \leq M\right\} .
$$

The space $X(M)$ is complete under the metric

$$
d((\psi, \zeta, \mathbf{T}),(\hat{\psi}, \hat{\zeta}, \hat{\mathbf{T}}))=\|\psi-\hat{\psi}\|_{4}+\|\zeta-\hat{\zeta}\|_{2}+\|\mathbf{T}-\hat{\mathbf{T}}\|_{2} .
$$

We choose $M$ small relative to 1 , but sufficiently large relative to the norms of the prescribed data. In order to prove the theorem, it is sufficient to show that the mapping defined by the iteration (12)-(18) is a contraction in $X(M)$. We begin by showing that the iteration maps $X(M)$ into itself. Let us assume that $\left(\psi^{n}, \zeta^{n}, \mathbf{T}^{n}\right)$ lies in $X(M)$.

We first discuss the solution of (13)-(15). A rearrangement of (15) yields

$$
\frac{\partial^{2}}{\partial x_{1} \partial x_{2}}\left(T_{22}^{n+1}-T_{11}^{n+1}\right)+\left(\frac{\partial^{2}}{\partial x_{1}^{2}}-\frac{\partial^{2}}{\partial x_{2}^{2}}\right) T_{12}^{n+1}=\rho \operatorname{curl}\left(\left(v^{n} \cdot \nabla\right) v^{n}\right)-\operatorname{curl} f .
$$

We can use (13) to express $x_{1}$-derivatives of the stresses, i.e.

$$
\frac{\partial}{\partial x_{1}} T_{i j}^{n+1}=\frac{1}{v_{1}^{n}}\left[-v_{2}^{n} \frac{\partial}{\partial x_{2}} T_{i j}^{n+1}-\lambda T_{i j}^{n+1} \quad \ldots\right] .
$$

After substituting (22) in (21), we obtain an ODE from which we can determine $T_{12}^{n+1}$ at the inflow boundary $x_{1}=0$. We denote this boundary value by $t^{n+1}$. The following estimate is immediate

$$
\left\|t^{n+1}\right\|_{\langle 3\rangle} \leq C\left(\left\|t_{1}\right\|_{\langle 3\rangle}+\left\|t_{2}\right\|_{\langle 3\rangle}+\left\|w^{n}\right\|_{4}+\left\|w^{n}\right\|_{4}\left\|\mathbf{T}^{n}\right\|_{3}+\left\|\mathbf{T}^{n}\right\|_{3}^{2}\right) .
$$

Here we have set $w^{n}=v^{n}-(V, 0)$. After determining $t^{n+1}$, we have a full set of initial conditions to solve (13). Using standard energy estimates for hyperbolic equations (see [4] for some more details) we obtain a unique solution which satisfies an estimate of the form

$$
\begin{gathered}
\left\|\mathbf{T}^{n+1}\right\|_{3,0}+\left\|\mathbf{T}^{n+1}\right\|_{2,1}+\left\|\mathbf{T}^{n+1}\right\|_{1,2}+\left\|\mathbf{T}^{n+1}\right\|_{0,3} \\
\leq C\left(\left\|t_{1}\right\|_{\langle 3\rangle}+\left\|t_{2}\right\|_{\langle 3\rangle}+\left\|t^{n+1}\right\|_{\langle 3\rangle}+\left\|w^{n}\right\|_{4}+\left\|\mathbf{T}^{n}\right\|_{3}\left\|w^{n}\right\|_{4}+\left\|\mathbf{T}^{n}\right\|_{3}^{2}\right) .
\end{gathered}
$$


From equation (13), we can see that an expression like the one on the right hand side of (24) also provides an upper bound for $\left\|\left(v^{n} \cdot \nabla\right) \mathbf{T}^{n+1}\right\|_{3}$.

For the solution of the initial-value problem (16), (17), one readily obtains the estimate

$$
\left\|\zeta^{n+1}\right\|_{0,2}+\left\|\zeta^{n+1}\right\|_{1,1}+\left\|\zeta^{n+1}\right\|_{2,0} \leq C\left(\left\|\zeta_{0}\right\|_{\langle 2\rangle}+\left\|\eta_{0}\right\|_{\langle 1\rangle}+\left\|r^{n}\right\|_{1,0}+\left\|r^{n}\right\|_{0,1}\right) .
$$

This is insufficient because we need to estimate third order derivatives of $\zeta^{n+1}$. Results available in the literature would require the existence of a higher order derivative of $r^{n}$ either with respect to $x_{1}$ or with respect to $x_{2}$. We cannot use such an assumption because of the dependence of $r$ on second derivatives of $\mathbf{T}^{n+1}$. However, because of the bound for $\left\|\left(v^{n} \cdot \nabla\right) \mathbf{T}^{n+1}\right\|_{3}$, we can get bounds on $\left\|\left(v^{n} \cdot \nabla\right) r^{n}\right\|_{1}$. Instead of differentiating (16) with respect to either $x_{1}$ or $x_{2}$, which is what is usually done, we can apply the operation $\left(v^{n} \cdot \nabla\right)$ to it. By doing this and deriving energy estimates in the usual fashion, we obtain an estimate of the form

$$
\|\zeta\|_{3} \leq C\left(\left\|\zeta_{0}\right\|_{\langle 3\rangle}+\left\|\eta_{0}\right\|_{\langle 2\rangle}+\left\|r^{n}\right\|_{0,1}+\left\|r^{n}\right\|_{1,0}+\left\|\left(v^{n} \cdot \nabla\right) r^{n}\right\|_{1} \cdot\right)
$$

By taking into account the form of $r$, we can estimate the last three terms in (26) by a constant times

$$
\|f\|_{4}+\left(\left\|w^{n}\right\|_{4}+\left\|\mathbf{T}^{n+1}\right\|_{3,0}+\left\|\mathbf{T}^{n+1}\right\|_{2,1}+\left\|\mathbf{T}^{n+1}\right\|_{1,2}+\left\|\mathbf{T}^{n+1}\right\|_{0,3}+\left\|\left(v^{n} \cdot \nabla\right) \mathbf{T}^{n+1}\right\|_{3}\right)^{2} .
$$

Finally, from (18) we immediately obtain

$$
\left\|\psi^{n+1}+V x_{2}\right\|_{5} \leq C\left(\left\|\psi_{0}\right\|_{\langle 9 / 2\rangle}+\left\|\psi_{1}\right\|_{\langle 9 / 2\rangle}+\left\|\zeta^{n+1}\right\|_{3}\right) .
$$

The claim that the iteration maps $X(M)$ into itself now follows easily by combining the estimates (23)-(28).

The derivation of estimates to show that the mapping defined by the iteration is a contraction is fairly straightforward, and we shall only demonstrate one step. From (16), (17) we obtain

$$
\begin{gathered}
\rho\left(\left(v^{n} \cdot \nabla\right)+\lambda\right)\left(v^{n} \cdot \nabla\right)\left(\zeta^{n+1}-\zeta^{n}\right)=\mu \Delta\left(\zeta^{n+1}-\zeta^{n}\right) \\
+\left(T_{k j}^{n+1}-P_{k j}\left(\mathbf{T}^{n+1}\right)\right) \frac{\partial^{2}\left(\zeta^{n+1}-\zeta^{n}\right)}{\partial x_{j} \partial x_{k}}-P_{22}\left(\mathbf{T}^{n+1}\right) \frac{\partial^{2}\left(\zeta^{n+1}-\zeta^{n}\right)}{\partial x_{1}^{2}} \\
-P_{11}\left(\mathbf{T}^{n+1}\right) \frac{\partial^{2}\left(\zeta^{n+1}-\zeta^{n}\right)}{\partial x_{2}^{2}}+\left(P_{12}\left(\mathbf{T}^{n+1}\right)+P_{21}\left(\mathbf{T}^{n+1}\right)\right) \frac{\partial^{2}\left(\zeta^{n+1}-\zeta^{n}\right)}{\partial x_{1} \partial x_{2}} \\
-\rho\left[\left(\left(v^{n} \cdot \nabla\right)+\lambda\right)\left(v^{n} \cdot \nabla\right)-\left(\left(v^{n-1} \cdot \nabla\right)+\lambda\right)\left(v^{n-1} \cdot \nabla\right)\right] \zeta^{n}
\end{gathered}
$$




$$
\begin{gathered}
+\left[\left(T_{k j}^{n+1}-P_{k j}\left(\mathbf{T}^{n+1}\right)\right)-\left(T_{k j}^{n}-P_{k j}\left(\mathbf{T}^{n}\right)\right)\right] \frac{\partial^{2} \zeta^{n}}{\partial x_{j} \partial x_{k}} \\
-\left[P_{22}\left(\mathbf{T}^{n+1}\right)-P_{22}\left(\mathbf{T}^{n}\right)\right] \frac{\partial^{2} \zeta^{n}}{\partial x_{1}^{2}}-\left[P_{11}\left(\mathbf{T}^{n+1}\right)-P_{11}\left(\mathbf{T}^{n}\right)\right] \frac{\partial^{2} \zeta^{n}}{\partial x_{2}^{2}} \\
+\left[\left(P_{12}\left(\mathbf{T}^{n+1}\right)+P_{21}\left(\mathbf{T}^{n+1}\right)\right)-\left(P_{12}\left(\mathbf{T}^{n}\right)+P_{21}\left(\mathbf{T}^{n}\right)\right)\right] \frac{\partial^{2} \zeta^{n}}{\partial x_{1} \partial x_{2}}+r^{n}-r^{n-1}, \\
\zeta^{n+1}-\zeta^{n}=0, \text { on } x_{1}=0, \frac{\partial\left(\zeta^{n+1}-\zeta^{n}\right)}{\partial x_{1}}=0, \text { on } x_{1}=0 .
\end{gathered}
$$

Energy estimates now yield

$$
\left\|\zeta^{n+1}-\zeta^{n}\right\|_{0,2}+\left\|\zeta^{n+1}-\zeta^{n}\right\|_{1,1}+\left\|\zeta^{n+1}-\zeta^{n}\right\|_{2,0}
$$

$$
\leq C\left(\left\|\zeta^{n}\right\|_{3}\left(\left\|v^{n}-v^{n-1}\right\|_{3}+\left\|\mathbf{T}^{n+1}-\mathbf{T}^{n}\right\|_{2}\right)+\left\|r^{n}-r^{n-1}\right\|_{0,0}+\left\|\left(v^{n} \cdot \nabla\right)\left(r^{n}-r^{n-1}\right)\right\|_{0}\right) .
$$

We note that a bound on $\left\|\zeta^{n}\right\|_{3}$ has already been obtained. In order to deal with the last term in (31), we note that

$$
\left(v^{n} \cdot \nabla\right)\left(r^{n}-r^{n-1}\right)=\left(v^{n} \cdot \nabla\right) r^{n}-\left(v^{n-1} \cdot \nabla\right) r^{n-1}+\left(\left(v^{n}-v^{n-1}\right) \cdot \nabla\right) r^{n-1} .
$$

By taking into account the form of $r$ and equation (13), it is easy to estimate the terms on the right hand side of (32).

3. Modifications in three dimensions. The basic iteration scheme used to construct solutions and the function spaces chosen for the analysis will be as in the twodimensional case, and we shall therefore confine the following discussion to those points where modifications are needed. One of these changes is that $\zeta=\operatorname{curl} v$ is now a vector, and the equation analogous to (9), written in components, is

$$
\begin{gathered}
\rho((v \cdot \nabla)+\lambda)(v \cdot \nabla) \zeta_{i}=\mu \Delta \zeta_{i}+\left(T_{k j}-P_{k j}(\mathbf{T})\right) \frac{\partial^{2} \zeta_{i}}{\partial x_{j} \partial x_{k}}-\epsilon_{m l i} \epsilon_{k r s} P_{m k}(\mathbf{T}) \frac{\partial^{2} \zeta_{s}}{\partial x_{l} \partial x_{r}} \\
+r_{i}\left(v, \nabla v, \nabla^{2} v, \mathbf{T}, \nabla \mathbf{T}, \nabla^{2} \mathbf{T}, f, \nabla f, \nabla^{2} f\right) .
\end{gathered}
$$

This is a system of PDE's for the components of $\zeta$, and in order to make it symmetric hyperbolic, we add the assumption that the matrix $\mathbf{P}$ is symmetric. In the iteration, we use the following equation which is analogous to (16):

$$
\rho\left(\left(v^{n} \cdot \nabla\right)+\lambda\right)\left(v^{n} \cdot \nabla\right) \zeta_{i}^{n+1}=\mu \Delta \zeta_{i}^{n+1}+\left(T_{k j}^{n+1}-P_{k j}\left(\mathbf{T}^{n+1}\right)\right) \frac{\partial^{2} \zeta_{i}^{n+1}}{\partial x_{j} \partial x_{k}}
$$




$$
\begin{gathered}
-\epsilon_{m l i} \epsilon_{k r s} P_{m k}\left(\mathbf{T}^{n+1}\right) \frac{\partial^{2} \zeta_{s}^{n+1}}{\partial x_{l} \partial x_{r}} \\
+r_{i}\left(v^{n}, \nabla v^{n}, \nabla^{2} v^{n}, \mathbf{T}^{n+1}, \nabla \mathbf{T}^{n+1}, \nabla^{2} \mathbf{T}^{n+1}, f, \nabla f, \nabla^{2} f\right) .
\end{gathered}
$$

The velocity can be determined in terms of the vorticity if we prescribe the normal velocity on both boundaries and the mean flux in the $y$ - and $z$-directions. Unfortunately, we shall not be able to guarantee that all the iterates satisfy $\operatorname{div} \zeta^{n+1}=0$, and hence we cannot simply use the equation $\operatorname{curl} v^{n+1}=\zeta^{n+1}$. Let $\Pi$ denote the orthogonal projection (in $L^{2}$ ) onto the subspace

$$
V=\left\{\zeta \in L^{2} \mid \operatorname{div} \zeta=0, \int_{0}^{L} \int_{0}^{M} \zeta_{1}\left(\cdot, x_{2}, x_{3}\right) d x_{3} d x_{2}=0\right\}
$$

The set of equations determining $v^{n+1}$ is

$$
\begin{gathered}
\operatorname{curl} v^{n+1}=\Pi \zeta^{n+1}, \operatorname{div} v^{n+1}=0 \\
v_{1}^{n+1}=V+a\left(x_{2}, x_{3}\right), \text { at } x_{1}=0, v_{1}^{n+1}=V+b\left(x_{2}, x_{3}\right) \text { at } x_{1}=1, \\
\int_{0}^{1} \int_{0}^{L} \int_{0}^{M} v_{2}^{n+1} d x_{3} d x_{2} d x_{1}=\alpha, \int_{0}^{1} \int_{0}^{L} \int_{0}^{M} v_{3}^{n+1} d x_{3} d x_{2} d x_{1}=\beta .
\end{gathered}
$$

Here the numbers $\alpha$ and $\beta$ as well as the functions $a$ and $b$ are prescribed and $|\alpha|+|\beta|+$ $\|a\|_{\langle 7 / 2\rangle}+\|b\|_{\langle 7 / 2\rangle}$ is assumed to be small. Moreover, we have to assume the compatibility condition

$$
\int_{0}^{L} \int_{0}^{M} a\left(x_{2}, x_{3}\right) d x_{3} d x_{2}=\int_{0}^{L} \int_{0}^{M} b\left(x_{2}, x_{3}\right) d x_{3} d x_{2} .
$$

It can easily be shown along the same lines as in Section 2 that by combining (34), (36) with (13) and (small) inflow data for $\mathbf{T}, \zeta$ and $\partial \zeta / \partial x_{1}$, we obtain a convergent iteration. However, there are two problems:

1. It is not guaranteed that the limit of the iteration satisfies $\zeta=\operatorname{curl} v$, or, equivalently, $\zeta=\Pi \zeta$.

2. It is not guaranteed that the original equation of motion (1) holds. This is because in proceeding from (1) to (6) we have applied the operation $(v \cdot \nabla)+\lambda+(\nabla v)^{T}$. In order to reverse this step and go from (6) to (1), we have to assume that (1) holds on the inflow boundary $x_{1}=0$ (cf. [4]). In two dimensions we imposed this condition as equation (15). 
In order to remove these two difficulties, we must restrict the inflow data; i.e., only part of these data can be prescribed, and the rest have to be determined at each step of the iteration.

We take the divergence of equation (33). If $\zeta$ were equal to curl $v$, we would get 0 (to see this, recall that (33) was derived by taking the curl of (6)). Hence we find (we set $\operatorname{curl} v=\omega)$

$$
\begin{gathered}
\frac{\partial}{\partial x_{i}}\left[\rho((v \cdot \nabla)+\lambda)(v \cdot \nabla)\left(\zeta_{i}-\omega_{i}\right)-\mu \Delta\left(\zeta_{i}-\omega_{i}\right)\right. \\
\left.-\left(T_{k j}-P_{k j}(\mathbf{T})\right) \frac{\partial^{2}\left(\zeta_{i}-\omega_{i}\right)}{\partial x_{j} \partial x_{k}}+\epsilon_{m l i} \epsilon_{k r s} P_{m k}(\mathbf{T}) \frac{\partial^{2}\left(\zeta_{s}-\omega_{s}\right)}{\partial x_{l} \partial x_{r}}\right]=0 .
\end{gathered}
$$

After some algebra, this yields

$$
\begin{gathered}
{\left[\rho((v \cdot \nabla)+\lambda)(v \cdot \nabla)-\mu \Delta-\left(T_{k j}-P_{k j}(\mathbf{T})\right) \frac{\partial^{2}}{\partial x_{j} \partial x_{k}}\right] \operatorname{div} \zeta} \\
=D^{2}\left(v, \nabla v, \nabla^{2} v, \mathbf{T}, \nabla \mathbf{T}\right)(\zeta-\omega) .
\end{gathered}
$$

Here $D^{2}$ is a second order differential operator with coefficients depending on the arguments indicated. Let $d_{1}$ denote the value of $\operatorname{div} \zeta$ at $x_{1}=0$, and let $d_{2}$ denote the value of $\frac{\partial}{\partial x_{1}} \operatorname{div} \zeta$ at $x_{1}=0$. From (39), we obtain the estimate

$$
\|\operatorname{div} \zeta\|_{0,1}+\|\operatorname{div} \zeta\|_{1,0} \leq C\left(\left\|d_{1}\right\|_{\langle 1\rangle}+\left\|d_{2}\right\|_{\langle 0\rangle}+\left(\|w\|_{4}+\|\mathbf{T}\|_{3}\right)\|\zeta-\omega\|_{2}\right) .
$$

As before, $w$ denotes $v-(V, 0,0)$. We note that $\|w\|_{4}+\|\mathbf{T}\|_{3}$ is small. Moreover, (36) yields the estimate

$$
\|\zeta-\omega\|_{2} \leq C\|\operatorname{div} \zeta\|_{1}+\left|\int_{0}^{L} \int_{0}^{M} \zeta_{1}\left(0, x_{2}, x_{3}\right) d x_{3} d x_{2}\right| .
$$

Inflow conditions are now handled as follows. We prescribe arbitrary data for $\zeta_{2}, \zeta_{3}$ and their normal derivatives. The initial datum for $\frac{\partial \zeta_{1}}{\partial x_{1}}$ is then determined by requiring that $\operatorname{div} \zeta=0$ at $x_{1}=0$. Finally, the initial datum for $\zeta_{1}$ cannot be determined a priori, but must be computed at each step of the iteration. We require that, at $x_{1}=0$,

$$
\frac{\partial^{2} \zeta_{1}^{n+1}}{\partial x_{1}^{2}}+\frac{\partial^{2} \zeta_{2}^{n+1}}{\partial x_{2} \partial x_{1}}+\frac{\partial^{2} \zeta_{3}^{n+1}}{\partial x_{3} \partial x_{1}}=s_{n+1}
$$

where $s_{n+1}$ is a constant to be determined. We then solve (42) for $\partial^{2} \zeta_{1}^{n+1} / \partial x_{1}^{2}$ and substitute into the first equation of (34). This yields an elliptic problem from which we 
can uniquely determine the inflow datum for $\zeta_{1}^{n+1}$ up to an arbitrary constant, as well as the constant $s_{n+1}$. Finally, the arbitrary constant in $\zeta_{1}^{n+1}$ is fixed by the requirement that

$$
\int_{0}^{L} \int_{0}^{M} \zeta_{1}^{n+1}\left(0, x_{2}, x_{3}\right) d x_{3} d x_{2}=0
$$

For the limit of the iteration, this obviously insures that $d_{1}=0, d_{2}=s$, and $\int_{0}^{L} \int_{0}^{M} \zeta_{1}\left(0, x_{2}, x_{3}\right) d x_{3} d x_{2}=0$. To determine the constant $s$, we take the first equation of (33), set $x_{1}=0$, and integrate over $x_{2}$ and $x_{3}$. If $\zeta$ is replaced by $\omega=$ curl v, we obtain an expression which vanishes identically (recall that (33) was derived by taking the curl of (6)). Hence we find

$$
\begin{gathered}
\int_{0}^{L} \int_{0}^{M}\left[\rho((v \cdot \nabla)+\lambda)(v \cdot \nabla)\left(\zeta_{1}-\omega_{1}\right)-\mu \Delta\left(\zeta_{1}-\omega_{1}\right)\right. \\
-\left(T_{k j}-P_{k j}(\mathrm{~T})\right) \frac{\partial^{2}\left(\zeta_{1}-\omega_{1}\right)}{\partial x_{j} \partial x_{k}} \\
\left.+\epsilon_{m l 1} \epsilon_{k r s} P_{m k}(\mathbf{T}) \frac{\partial^{2}\left(\zeta_{s}-\omega_{s}\right)}{\partial x_{l} \partial x_{r}}\right]\left(0, x_{2}, x_{3}\right) d x_{3} d x_{2}=0 .
\end{gathered}
$$

Next we integrate by parts in all terms which involve second order derivatives of $\zeta-\omega$ such that one of the differentiations is with respect to $x_{2}$ or $x_{3}$. This leads to terms which can be estimated by a constant times $\left(\|\mathbf{T}\|_{3}+\|w\|_{4}\right)\|\zeta-\omega\|_{2}$. The term which remains is the integral of

$$
\begin{gathered}
\rho v_{1}^{2} \frac{\partial^{2}}{\partial x_{1}^{2}}\left(\zeta_{1}-\omega_{1}\right)+\lambda \rho v_{1} \frac{\partial}{\partial x_{1}}\left(\zeta_{1}-\omega_{1}\right)-\mu \frac{\partial^{2}}{\partial x_{1}^{2}}\left(\zeta_{1}-\omega_{1}\right) \\
-\left(T_{11}-P_{11}(\mathbf{T})\right) \frac{\partial^{2}}{\partial x_{1}^{2}}\left(\zeta_{1}-\omega_{1}\right)
\end{gathered}
$$

We now note that

$$
\begin{gathered}
\frac{\partial}{\partial x_{1}}\left(\zeta_{1}-\omega_{1}\right)=-\frac{\partial}{\partial x_{2}}\left(\zeta_{2}-\omega_{2}\right)-\frac{\partial}{\partial x_{3}}\left(\zeta_{3}-\omega_{3}\right), \\
\frac{\partial^{2}}{\partial x_{1}^{2}}\left(\zeta_{1}-\omega_{1}\right)=-\frac{\partial^{2}}{\partial x_{2} \partial x_{1}}\left(\zeta_{2}-\omega_{2}\right)-\frac{\partial^{2}}{\partial x_{3} \partial x_{3}}\left(\zeta_{3}-\omega_{3}\right)+s .
\end{gathered}
$$

By using this, we obtain again terms which can, after an integration by parts, be estimated by a constant times $\left(\|\mathbf{T}\|_{3}+\|w\|_{4}\right)\|\zeta-\omega\|_{2}$, plus $s$ times the integral of $\rho v_{1}^{2}-\mu-T_{11}+P_{11}(\mathbf{T})$. 
As a result, $s$ can be estimated by a constant times $\left(\|\mathbf{T}\|_{3}+\|w\|_{4}\right)\|\zeta-\omega\|_{2}$. In conjunction with (40) and (41) this yields that $\operatorname{div} \zeta$ is indeed zero.

To make sure that (1) is satisfied, we proceed as in [4]. At each step of the iteration, $q^{n+1}$ is determined by the relation

$$
\nabla q^{n+1}=\Sigma\left[\left(\left(v^{n} \cdot \nabla\right)+\lambda+\left(\nabla v^{n}\right)^{T}\right)\left(\operatorname{div} \mathbf{T}^{n}+f-\rho\left(v^{n} \cdot \nabla\right) v^{n}\right)\right] .
$$

Here $\Sigma$ is the orthogonal projection of $L^{2}$ onto the subspace of vectorfields with vanishing curl. From (47), $q^{n+1}$ is determined up to an arbitrary constant; we may fix this constant by requiring that

$$
\int_{0}^{1} \int_{-L / 2}^{L / 2} \int_{-M / 2}^{M / 2} q^{n+1}\left(x_{1}, x_{2}, x_{3}\right) d x_{3} d x_{2} d x_{1}=0 .
$$

We note that $q^{n+1}$ is not necessarily periodic in the $x_{2}$ and $x_{3}$-directions, but may contain a part which is linear in $x_{2}$ and $x_{3}$. At $x_{1}=0$, we impose the condition

$$
\rho\left(v^{n} \cdot \nabla\right) v^{n}=\operatorname{div} \mathbf{T}^{n+1}-\nabla p^{n+1}+f .
$$

This condition, in conjunction with (13) and the equation

$$
\left(v^{n} \cdot \nabla\right) p^{n+1}+\lambda p^{n+1}=q^{n+1}
$$

can be used to express some of the inflow data for $\mathbf{T}$ in terms of others. For details we refer to [4]. Specifically we can prescribe the stress components specified in (5) and solve for the rest.

Let us summarize the iteration scheme. We prescribe the following boundary data a priori: the normal velocities on both boundaries and the total flux in the $x_{2}$ - and $x_{3}$ directions according to (35); the second and third components of the vorticity and their normal derivatives at the inflow boundary,

$$
\begin{gathered}
\zeta_{2}\left(0, x_{2}, x_{3}\right)=\zeta_{2}^{0}\left(x_{2}, x_{3}\right), \zeta_{3}\left(0, x_{2}, x_{3}\right)=\zeta_{3}^{0}\left(x_{2}, x_{3}\right), \\
\frac{\partial \zeta_{2}}{\partial x_{1}}\left(0, x_{2}, x_{3}\right)=\zeta_{2}^{1}\left(x_{2}, x_{3}\right), \frac{\partial \zeta_{3}}{\partial x_{1}}\left(0, x_{2}, x_{3}\right)=\zeta_{3}^{1}\left(x_{2}, x_{3}\right), \\
\frac{\partial \zeta_{1}}{\partial x_{1}}\left(0, x_{2}, x_{3}\right)=-\frac{\partial \zeta_{2}^{0}}{\partial x_{2}}\left(x_{2}, x_{3}\right)-\frac{\partial \zeta_{3}^{0}}{\partial x_{3}}\left(x_{2}, x_{3}\right) ;
\end{gathered}
$$

and the inflow stresses according to (5). We denote this prescribed part of the stress by $\mathbf{T}_{p}$. We start the iteration by setting $\mathbf{T}=0, \zeta=0, v=(V, 0,0)$. At each step of the iteration, we first determine $q^{n+1}$ from (47), (48). Then we calculate the inflow boundary value of $\mathbf{T}^{n+1}$ from (5), (49), (50) and (13). We can now determine $\mathbf{T}^{n+1}$ from (13). Next we use (42), (43) and (34) to determine the inflow value of $\zeta_{1}^{n+1}$. Then we determine $\zeta^{n+1}$ from (34) and $v^{n+1}$ from (36).

The existence theorem thus obtained is the following: 
Theorem:. Assume that $\|f\|_{4},\|a\|_{\langle 7 / 2\rangle},\|b\|_{\langle 7 / 2\rangle},\left\|\zeta_{2}^{0}\right\|_{\langle 3\rangle},\left\|\zeta_{3}^{0}\right\|_{\langle 3\rangle},\left\|\zeta_{2}^{1}\right\|_{\langle 2\rangle},\left\|\zeta_{3}^{1}\right\|_{\langle 2\rangle}$, $\left\|\mathbf{T}_{p}\right\|_{\langle 3\rangle},|\alpha|$ and $|\beta|$ are sufficiently small. Assume, moreover, that the matrix function $\mathbf{P}$ has symmetric values. Then there is a solution of (1-3) which satisfies the boundary conditions given by (5), (35) and (51) and the regularity $v \in H^{4}, \mathbf{T} \in H^{3}$. Moreover, this solution is the only one for which $\|v-(V, 0,0)\|_{4}$ and $\|\mathbf{T}\|_{3}$ are small.

\section{REFERENCES}

[1] D.D. Joseph, M. Renardy and J.C. Saut, Hyperbolicity and change of type in the flow of viscoelastic fluids, Arch. Rat. Mech. Anal., 87 (1985), pp. 213-251.

[2] M. Luskin, On the classification of some model equations for viscoelasticity, J. Non-Newt. Fluid Mech., 16 (1984), pp. 3-11.

[3] J.G. Oldroyd, Non-Newtonian effects in steady motion of some idealized elastico-viscous liquids, Proc. Roy. Soc. London, A 245 (1958), pp. 278-297.

[4] M. Renardy, Inflow boundary conditions for steady flow of viscoelastic fluids with differential constitutive laws, Rocky Mt. J. Math., 18 (1988), pp. 445-453.

[5] Recent advances in the mathematical theory of steady flows of viscoelastic fluids, J. NonNewt. Fluid Mech., 29 (1988), pp. 11-24.

[6] Boundary conditions for steady flows of non-Newtonian fluids, Proc. Xth Int. Congr. Rheology (ed. P.H.T. Uhlherr), Vol. 2, Sydney, 1988, pp. 202-204.

[7] I.M. RUTKEvich, The propagation of small perturbations in a viscoelastic fluid, J. Appl. Math. Mech., 34 (1970), pp. 35-50.

[8] J.S. Ultman and M.M. Denn, Anomalous heat transfer and a wave phenomenon in dilute polymer solutions, Trans. Soc. Rheol., 14 (1970), pp. 307-317. 


\section{Recent IMA Preprints}

Title

Yisong Yang Existence, Regularity, and Asymptotic Behavior of the Solutions to the Ginzburg-Landau Equations on $\mathbf{R}^{3}$

Chjan. C. Lim On Symplectic Tree Graphs

Wilhelm I. Fushchich, Ivan Krivsky and Vladimir Simulik, On Vector and Pseudovector Lagrangians for Electromagnetic Field

Wilhelm I. Fushchich, Exact Solutions of Multidimensional Nonlinear Dirac's and Schrödinger's Equations

Wilhelm I. Fushchich and Renat Zhdanov, On Some New Exact Solutions of Nonlinear D'Allembert and Hamilton Equations

Brian A. Coomes, The Lorenz System Does Not Have a Polynomial Flow

J.W. Helton and N.J. Young, Approximation of Hankel Operators: Truncation Error in an $H^{\infty}$ Design Method

Gregory Ammar and Paul Gader, A Variant of the Gohberg-Semencul Formula Involving Circulant Matrices

R.L. Fosdick and G.P. MacSithigh, Minimization in Nonlinear Elasticity Theory for Bodies Reinforced with Inextensible Cords

Fernando Reitich, Rapidly Stretching Plastic Jets: The Linearized Problem

Francisco Bernis and Avner Friedman, Higher Order Nonlinear Degenerate Parabolic Equations

Xinfu Chen and Avner Friedman, Maxwell's Equations in a Periodic Structure

Avner Friedman and Michael Vogelius Determining Cracks by Boundary Measurements

Yuji Kodama and John Gibbons, A Method for Solving the Dispersionless KP Hierarchy and its Exact Solutions II

Yuji Kodama, Exact Solutions of Hydrodynamic Type Equations Having Infinitely Many Conserved Densities

Robert Carroll, Some Forced Nonlinear Equations and the Time Evolution of Spectral Data

Chjan. C. Lim Spanning Binary Trees, Symplectic Matrices, and Canonical Transformations for Classical N-body Problems

E.F. Assmus, Jr. and J.D. Key, Translation Planes and Derivation Sets

Matthew Witten, Mathematical Modeling and Computer Simulation of the Aging-Cancer Interface

Matthew Witten and Caleb E. Finch, Re-Examining The Gompertzian Model of Aging

Bei Hu, A Free Boundary Problem for a Hamilton-Jacobi Equation Arising in Ions Etching

T.C. Hu, Victor Klee and David Larman, Optimization of Globally Convex Functions

Pierre Goossens, Shellings of Tilings

D. David, D. D. Holm, and M.V. Tratnik, Integrable and Chaotic Polarization Dynamics in Nonlinear Optical Beams

D. David, D.D. Holm and M.V. Tratnik, Horseshoe Chaos in a Periodically Perturbed Polarized Optical Beam

Laurent Habsieger, Linear Recurrent Sequences and Irrationality Measures

Laurent Habsieger, MacDonald Conjectures and The Selberg Integral

David Kinderlehrer and Giorgio Vergara-Caffarelli, The Relaxation of Functionals with Surface Energies

Richard James and David Kinderlehrer, Theory of Diffusionless Phase Transitions

David Kinderlehrer, Recent Developments in Liquid Crystal Theory

Niky Kamran and Peter J. Olver, Equivalence of Higher Order Lagrangians

1. Formulation and Reduction

Lucas Hsu, Niky Kamran and Peter J. Olver, Equivalence of Higher Order Lagrangians II. The Cartan Form for Particle Lagrangians

D.J. Kaup and Peter J. Olver, Quantization of BiHamiltonian Systems

Metin Arik, Fahrünisa Neyzi, Yavuz Nutku, Peter J. Olver and John M. Verosky Multi-Hamiltonian Structure of the Born-Infeld Equation

David H. Wagner, Detonation Waves and Deflagration Waves in the One Dimensional ZND Model for High Mach Number Combustion

Jerrold R. Griggs and Daniel J. Kleitman, Minimum Cutsets for an Element of a Boolean Lattice

Dieter Jungnickel, On Affine Difference Sets

Pierre Leroux, Reduced Matrices and q-log Concavity Properties of q-Stirling Numbers

A. Narain and Y. Kizilyalli, The Flow of Pure Vapor Undergoing Film Condensation Between Parallel Plates

Donald A. French, On the Convergence of Finite Element Approximations of a Relaxed Variational Problem 
Yisong Yang, Computation, Dimensionality, and Zero Dissipation Limit of the Ginzburg-Landau Wave Equation

Jürgen Sprekels, One-Dimensional Thermomechanical Phase Transitions with Non-Convex Potentials of Ginzburg-Landau Type

Yisong Yang, A Note On Nonabelian Vortices

Yisong Yang, On the Abelian Higgs Models with Sources

Chjan. C. Lim, Existence of Kam Tori in the Phase Space of Vortex Systems

John Weiss, Bäcklund Transformations and the Painlevé Property

Pu Fu-cho and D.H. Sattinger, The Yang-Baxter Equation for Integrable Systems

E. Bruce Pitman and David G. Schaeffer, Instability and Ill-Posedness in Granular Flow

Brian A. Coomes, Polynomial Flows on $\mathrm{C}^{n *}$

Bernardo Cockburn, Suchung Hou and Chi-Wang Shu, The Runge-Kutta Local Projection Discontinuous Galerkin Finite Element Method for Conservation Laws IV: The Multidimensional Case

Peter J. Olver, Invariant Theory, Equivalence Problems, and the Calculus of Variations Daniel D. Joseph and Thomas S. Lundgren with an appendix by R. Jackson and D.A. Saville, Ensemble Averaged and Mixture Theory Equations

P. Singh, Ph. Caussignac, A. Fortes, D.D. Joseph and T. Lundgren, Stability of Periodic Arrays of Cylinders Across the Stream by Direct Simulation

Daniel D. Joseph, Generalization of the Foscolo-Gibilaro Analysis of Dynamic Waves

A. Narain and D.D. Joseph, Note on the Balance of Energy at a Phase Change Interface

Daniel D. Joseph, Remarks on inertial radii, persistent normal stresses, secondary motions, and non-elastic extensional viscosities

D. D. Joseph, Mathematical Problems Associated with the Elasticity of Liquids

Henry C. Simpson and Scott J. Spector, Some Necessary Conditions at an Internal Boundary for Minimizers in Finite Elasticity

Peter Gritzmann and Victor Klee, On the 0-1 Maximization of Positive Definite Quadratic Forms

Fu-Cho Pu and D.H. Sattinger, The Yang-Baxter Equations and Differential Identities

Avner Friedman and Fernando Reitich, A Hyperbolic Inverse Problem Arising in the Evolution of Combustion Aerosol

E.G. Kalnins, Raphael D. Levine and Willand Miller, Jr., Conformal Symmetries and Generalized Recurrences for Heat and Schrödinger Equations in One Spatial Dimension

Wang Jinghua and Gerald Warnecke, On Entropy Consistency of Large Time Step Godunov and Glimm Schemes

C. Guillopé and J.C. Saut, Existence Results for the Flow of Viscoelastic Fluids with a Differential Constitutive Law

H.L. Bodlaender, P. Gritzmann, V. Klee and J. Van Leeuwen Computational Complexity of Norm-Maximization

Li Ta-tsien (Li Da-qian) and Yu Xin, Life-Span of Classical Solutions to Fully Nonlinear Wave Equations

Jong-Shenq Guo, A Variational Inequality Associated with a Lubrication Problem

Jong-Shenq Guo, On the Semilinear Elliptic Equation $\Delta w-\frac{1}{2} y \cdot \nabla w+\lambda w-w^{-\beta}$ in $R^{n}$

Andrew E. Yagle, Inversion of the Bloch transform in magnetic resonance imaging using asymmetric two-component inverse scattering

Bei Hu, A Fiber Tapering Problem

Peter J. Olver, Canonical Variables for BiHamiltonian Systems

Michael Renardy, A Well-Posed Boundary Value Problem for Supercritical Flow of Viscoelastic Fluids of Maxwell Type

Michael Renardy, Ill-Posedness Resulting from Slip As a Possible Explanation of Melt Fracture

Michael Renardy, Compatibility Conditions at Corners Between Walls and Inflow Boundaries for Fluids of Maxwell Type

Rolf Rees, The Spectrum of Restricted Resolvable Designs with $r=2$

D. Lewis and J.C. Simo, Nonlinear stability of rotating pseudo-rigid bodies

Robert Hardt and David Kinderlehrer, Variational Principles with Linear Growth

San Yih Lin and Yisong Yang, Computation of Superconductivity in Thin Films

A. Narain, Pressure Driven Flow of Pure Vapor Undergoing Laminar Film Condensation Between Parallel Plates 